\title{
DIMENSIONAL REGULARIZATION AND THE TWO-LOOP AXIAL ANOMALY IN ABELIAN, NON-ABELIAN AND SUPERSYMMETRIC GAUGE THEORIES
}

\author{
D.R.T. JONES and J.P. LEVEILLE
}

The Randall Laboratory of Physics, The University of Michigan, Ann Arbor, MI 48109, USA

Received 12 January 1982

(Revised 19 April 1982)

\begin{abstract}
The two-loop corrections to the axial anomaly are calculated for a non-abelian gauge theory with fermions using both conventional and supersymmetric dimensional regularization. In both cases we find results consistent with the Adler-Bardeen theorem if we use the non-anticommuting $\gamma^{5}$ of 't Hooft and Veltman. Expectations (based on the supermultiplet structure of the anomalies) that there exists in $N=1$ supersymmetric Yang-Mills theory an axial current $J^{5}$ such that $\partial \cdot J^{5} \sim \beta(g) F \dot{F}$ are discussed.
\end{abstract}

\section{Introduction}

In this paper we present a calculation of the two-loop correction to the divergence of the axial current $\partial^{\mu} J_{\mu}^{S}$ in a non-abelian gauge theory with a multiplet of fermion fields transforming according to an arbitrary representation $\mathrm{R}$ of the gauge group. For simplicity we take the fermions to be massless throughout, so that chiral symmetry is broken only by the Adler-Bell-Jackiw (ABJ) anomaly. All our calculations are performed using dimensional regularization. One purpose of this paper is to investigate whether or not the well-known difficulties associated with $\gamma^{5}$ in dimensional regularization schemes can be dealt with, and to explicitly verify the Adler-Bardeen theorem at the two-loop level. For Majorana fermions transforming according to the adjoint representation we have $N=1$ supersymmetric Yang-Mills theory (SSYM) (in the Wess-Zumino gauge), and this special case provides us with additional motivation. As is well known [1-5] in SSYM the axial, the trace of the energy-momentum tensor and supersymmetry $(\gamma \cdot S)$ anomalies form the $F, G, \psi$ components respectively of a chiral scalar supermultiplet $(A B \psi F G)$. The leading contributions to the anomaly preserve the supermultiplet structure of the anomalies (as long as we use a regularization procedure for which the supersymmetry anomaly resides in $\gamma \cdot S$ rather than $\left.\partial_{\mu} S^{\mu}\right)[3,6]$. It is known that the trace anomaly is proportional to the Callan-Symanzik function $\beta(g)$ to all orders [7], while the Adler-Bardeen (AB) theorem [8] asserts that there are no radiative corrections to the 
axial anomaly beyond the fermion triangle. The most obvious resolution of this dilemma is that the $\mathrm{AB}$ theorem is simply not valid in supersymmetric theories; there might be a conflict between the proofs of the theorem and the additional constraints of the supersymmetry Ward identities. Since, however, the supermultiplet identification of the usual axial current in SSYM remains unclear (see for example the recent analysis of Piguet and Sibold [9]) we feel an explicit calculation may be useful. The results are given in ref. [10]; in this paper we give more details of the calculation and discussion of related issues. The Adler-Bardeen theorem in non-supersymmetric non-abelian gauge theories is considered to be established [11]. The only explicit calculation to verify this statement of which we are aware is that of Chanowitz [12]. He calculated the one-loop corrections to the operator $F_{\mu \nu} \tilde{F}^{\mu \nu}\left(\tilde{F}^{\mu \nu} \equiv \varepsilon^{\mu \nu \rho \lambda} F_{\rho \lambda}\right)$ in the spontaneously broken phase of an $\mathrm{SU}(2)$ gauge theory, using the modification of dimensional regularization proposed by Bardeen [11], and found results consistent with the $\mathrm{AB}$ theorem. Our motivation being somewhat different, we do not introduce breaking of the gauge symmetry and calculate the two-loop corrections of $\partial^{\mu} J_{\mu}^{5}$ as well as the one-loop corrections to $F^{\mu \nu} \tilde{F}_{\mu \nu}$. We perform the calculations using two regularization prescriptions: conventional dimensional regularization (CDR) [13-15] and supersymmetric dimensional regularization, or regularization by dimensional reduction (SDR) [16]. This choice of procedures is motivated by the fact that dimensional regularization (in some form) is the most practical way of performing explicit calculations beyond the one-loop level. The trade-off for the convenience of dimensional regularization is the difficulty in treating $\gamma^{5}$. We shall see, however, that if one uses the non-anticommuting $\gamma^{5}$ of 't Hooft and Veltman [13] and carefully imposes relevant Ward identities, then one obtains results consistent with the $A B$ theorem for both CDR and SDR in the abelian and the non-abelian case. As emphasized by Chanowitz et al. [17], use of a non-anticommuting $\gamma^{5}$ is inconvenient and can be avoided at the one-loop level. The reader is referred to ref. [17] for a discussion of their prescription and its possible extension to higher order.

In the model under consideration the axial current is not gauged so the anomaly has no implications concerning renormalizability. In currently popular theories of electroweak interactions there are indeed gauged axial currents. Renormalizability is maintained by choosing particle representations so that the axial anomalies cancel at one loop, and appealing to the $A B$ theorem to extend this result to all orders. $O$ f course, in a supersymmetric version of such a theory it is not at all clear whether the supermultiplet structure would place any restriction on the gauged axial currents; however, the question of whether supersymmetry implies non-vanishing higher order corrections to the anomalies of these currents clearly deserves further investigation. If the answer were in the affirmative the consequences for recently fashionable supersymmetric grand unification would be severe.

The rest of this paper is organized as follows. In the next section we review the status and limitations of the SDR method and discuss the treatment of $\gamma^{5}$ in the context of dimensional regularization. Sect. 3 is devoted to a discussion of the 
one-loop correction to $\partial \cdot J^{5}$. In sect. 4 we calculate the two-loop corrections to $\partial \cdot J^{5}$ and the one-loop corrections to $F \tilde{F}$, and compare the results with our expectations (based on supersymmetry) for the special case of $N=1$ supersymmetric Yang-Mills theory. In sect. 5 we present conclusions and speculations. In appendix $A$ an identity used in sect. 3 is derived, and in appendix B we describe the calculation of the two-loop Feynman integrals, and give results for some of the integrals that were encountered.

\section{Supersymmetric dimensional regularization}

Calculations beyond the tree approximation in field theories require a regularization method to deal with the resulting infinities. The method almost universally adopted in gauge theories is that of dimensional regularization [13-15], and for excellent reasons; it is manifestly gauge invariant, and convenient in practice. It was therefore natural to seek to apply dimensional regularization to supersymmetric theories. The difficulty that then arises is that supersymmetric theories are not invariant with respect to the supersymmetry transformations in a general number $n$ of space-time dimensions. The essential source of this problem is that a necessary condition for supersymmetry is equality of Bose and Fermi degrees of freedom; an equality which will hold only for specific values of $n$.

A possible solution to this problem retaining the benefits of conventional dimensional regularization (CDR) was proposed by Siegel [16]. The essential difference between Siegel's method, which we will call supersymmetric dimensional regularization (SDR), and CDR is that the continuation from 4 to $n$ dimensions is performed by dimensional reduction. Subsequent calculations confirmed that the method respected supersymmetric Ward identities at the one-loop level in both global [18] and local [19] supersymmetry (while CDR did not). It was subsequently pointed out, however, that at sufficiently high order the method fails to respect supersymmetry for general $n[20,21]$. The essential reason for this is that in SDR it is necessary to separate all 4 -vectors into $n$-dimensional and $(4-n)$-dimensional parts. Thus the decomposition

$$
\gamma_{\mu}=\gamma_{\hat{\mu}}+\gamma_{\tilde{\mu}}
$$

is assumed (formally) to exist for the $\gamma$ matrices. [In eq. (2.1) $\mu, \hat{\mu}$ and $\tilde{\mu}$ denote $4-, n$ and $(4-n)$-dimensional vector indices, respectively.] Consider the lagrangian for supersymmetric Yang-Mills theory $(N=1)$ (in the Wess-Zumino gauge)

$$
\mathcal{E}=-\frac{1}{4} G_{\mu \nu}^{2}+\frac{1}{2} i \bar{\psi} \gamma^{\mu} D_{\mu} \psi
$$


The variation $\delta \mathscr{E}$ of $E$ with respect to a supersymmetry transformation

$$
\begin{aligned}
& \delta A_{\mu}^{a}=i \bar{\varepsilon} \gamma_{\mu} \psi^{a}, \\
& \delta \psi^{a}=F_{\mu \nu}^{a} \sigma^{\mu v} \varepsilon,
\end{aligned}
$$

is (up to a total derivative)

$$
\delta \varrho=\frac{1}{2} g f^{a b c} \bar{\varepsilon} \gamma_{\mu} \psi^{a} \bar{\psi}^{b} \gamma^{\mu} \psi^{c} .
$$

$\delta \mathcal{E}$ can be shown to be zero by a Fierz rearrangement in 4-dimensional Minkowski space, but this Fierz rearrangement depends on properties of the $\gamma$ matrices inconsistent with the decomposition (2.1) for non-integer $n$. It was argued in ref. [21] that, for example, the supersymmetric Ward identity for the two-point function examined in ref. [18], may receive a non-vanishing contribution from $\delta \mathscr{L}$ at the 4-loop level. Thus it is still an open question as to whether there exists a modification of dimensional regularization which is fully supersymmetric. It is easily seen, however, that $\delta e$ will not contribute in our calculations at the one- or two-loop level. The use of a regularization method which fails to respect the underlying symmetries is, of course, fundamentally unsatisfactory, but, as we have already stated, it is difficult (for the authors at any rate) to contemplate such calculations without dimensional regularization (in some form).

We turn now to the treatment of $\gamma^{5}$. It was recognized in the earliest applications of dimensional regularization $[13,14]$ that $\gamma^{5}$ presents difficulties. These arise because a fully anticommuting $\gamma^{5}$ in $n$ dimensions implies immediately:

$$
n(n-2)(n-4) \operatorname{Tr}\left[\gamma^{5} \gamma_{\alpha} \gamma_{\beta} \gamma_{\gamma} \gamma_{\delta}\right]=0
$$

In their initial work on dimensional regularization 't Hooft and Veltman [13] recognized this problem and showed that a successful treatment of the one-loop anomaly was obtained by use of a $\gamma^{5}$ with the following properties:

$$
\begin{aligned}
& \left\{\gamma^{5}, \gamma_{\mu}\right\}=0, \\
& \left\{\gamma^{5}, \gamma_{\hat{\mu}}\right\}=2 \gamma^{5} \gamma_{\tilde{\mu}}=2 \gamma_{\tilde{\mu}} \gamma^{5},
\end{aligned}
$$

where $\delta_{\mu \mu}=4, \delta_{\hat{\mu} \hat{\mu}}=n$ and $\delta_{\tilde{\mu} \tilde{\mu}}=n-4$.

Consistent use of eq. (2.5) leads, of course, to spurious anomalies in other (non-anomalous) Ward identities. Subsequent authors [22-24] argued that these spurious anomalies could be dealt with, essentially by imposing the Ward identities on the renormalized Green functions. This procedure is unwieldy for diagrams involving even numbers of $\gamma^{5}$ s but poses no problems of principle. 
An alternative prescription which permits the use of an anticommuting $\gamma^{5}$ (at the one-loop level) has been given by Chanowitz et al. [17]; we will not discuss this method as it is unclear (to us) how it can be extended to higher orders.

Our procedure is therefore as follows: We write down all fermion loops starting with the $\gamma^{5}$, and then perform Feynman integrals and Dirac algebra without assuming any property of $\gamma^{5}$ at all, except that

$$
\operatorname{Tr}\left[\gamma^{5} \gamma^{\alpha} \gamma^{\beta}\right]=0
$$

This prescription is sufficient to enable us to achieve expressions which have finite limits as $n \rightarrow 4$. After this limit the usual formula

$$
\operatorname{Tr}\left[\gamma^{5} \gamma_{\alpha} \gamma_{\beta} \gamma_{\gamma} \gamma_{\delta}\right]=4 i \varepsilon_{\alpha \beta \gamma \delta}
$$

is used.

That this procedure is satisfactory for the evaluation of the lowest order VVA triangle has been noted by a number of authors [17,6,25]; we will for clarity reconsider this calculation and then follow the same procedure at the two-loop level.

We have verified that this procedure gives automatic vector current conservation at both one- and two-loop levels in QED. The usual ambiguity in the position of the anomaly corresponds to the arbitrariness involved in the choice of initial position for the $\gamma^{5}$.

We shall see, however, that in one graph (fig. $2 r$ ) we are obliged to use an explicit prescription for $\gamma^{5}$, in order to ensure that a Ward identity is obeyed. As we shall see, it is then eq. (2.5) which leads correctly to the Adler-Bardeen theorem. The calculation of fig. $2 \mathrm{r}$ will be considered in more detail in sect. 4 .

It has been argued that a non-anticommuting $\gamma^{5}$ such as that of eq. (2.5) is inconsistent with supersymmetry [26,6]. Certainly in the Wess-Zumino model, for example, the action is no longer invariant under the supersymmetry transformations and consequently spurious anomalies are generated, which must be dealt with as described above. The impact of these observations on our calculation is not clear, however, since $\partial \cdot J^{5}$ and $F \tilde{F}$ are not members of the same supermultiplet but rather equivalent (presumably) members of a different supermultiplet. It might also be objected that SDR, being based on dimensional reduction, definitely requires $n<4$, whereas the 't Hooft-Veltman $\gamma^{5}$ requires (formally, at least) $n>4$. We will return to these points in sect. 4 .

\section{Supersymmetry and the VVA triangle}

According to the Adler-Bardeen theorem, the divergence of the axial current $\bar{\psi} \gamma_{\mu} \gamma^{5} \psi$ is given in both abelian and non-abelian gauge theories by the expression

$$
\partial \cdot J^{5}=\frac{g^{2}}{16 \pi^{2}} T(\mathrm{R}) F \tilde{F}
$$


where $T(\mathrm{R})$ is given by

$$
T(\mathrm{R}) \delta^{a b}=\operatorname{Tr}\left[R^{a} R^{b}\right]
$$

for fermions transforming according to the representation $\mathrm{R}$.

In $N=1$ supersymmetric Yang-Mills theory (SSYM) however, (corresponding to the case of Majorana fermions in the adjoint representation) it has been conjectured that

$$
\partial \cdot J^{5}=\frac{1}{6} \frac{\beta(g)}{g} F \tilde{F} .
$$

The basis for eq. (3.2) is the statement that $\partial \cdot J^{5}$ forms a supermultiplet with $\theta_{\mu}^{\mu}$ and $\gamma \cdot S$. Then if one accepts that the relation $\theta_{\mu}^{\mu} \sim \beta(g) F^{2}$ is true in the supersymmetric case, it follows that eq. (3.2) rather than (3.1) is necessary if the supermultiplet nature of the currents is to be preserved.

Our purpose is to investigate the validity of eq. (3.1) [and in SSYM eq. (3.2)] to $O\left(g^{4}\right)$, by calculating the Green functions

$$
\begin{array}{r}
\left\langle 0\left|\mathrm{~T}\left(\partial \cdot J^{5}(x) A_{\mu}(y) A_{\nu}(z)\right)\right| 0\right\rangle, \\
\left\langle 0\left|\mathrm{~T}\left(F \tilde{F} A_{\mu}(y) A_{\nu}(z)\right)\right| 0\right\rangle .
\end{array}
$$

In momentum space

$$
\begin{gathered}
\left\langle 0\left|\partial \cdot J^{5} A_{\mu}\left(p_{1}\right) A_{\nu}\left(p_{2}\right)\right| 0\right\rangle=\left[\frac{A g^{2}}{16 \pi^{2}}+\frac{B g^{4}}{\left(16 \pi^{2}\right)^{2}}\right] \cdot 4 \varepsilon_{\mu \nu \rho \sigma} p_{1}^{\rho} p_{2}^{\sigma}, \\
\left\langle 0\left|F \tilde{F} A_{\mu}\left(p_{1}\right) A_{\nu}\left(p_{2}\right)\right| 0\right\rangle=2\left[1+\frac{C g^{2}}{16 \pi^{2}}\right] \cdot 4 \varepsilon_{\mu \nu \rho \sigma} p_{1}^{\rho} p_{2}^{\sigma} .
\end{gathered}
$$

Our aim is to calculate $A, B$ and $C$. In this section we consider the calculation of $A$. Of course, this calculation is nothing but the standard VVA triangle and has been performed already (using dimensional regularization) a number of times; nevertheless for completeness we include it here. First we show how a direct evaluation of the VVA triangle ${ }^{\star}$ leads to a determination of $A$; then we give a simplified derivation which readily extends to the two-loop level (the calculation of $B$ ) avoiding completely the necessity for studying unwieldy Feynman parameter integrals.

$\star$ We follow closely the derivation of Capper [25]. 


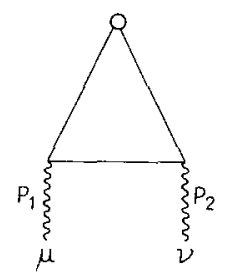

Fig. 1. The basic triangle diagram for the axial anomaly. The circle denotes a $\gamma^{5} \gamma^{\alpha}$ insertion.

The one-loop expression for the Green function (see fig. 1) $\langle 0| J_{\lambda}^{5}\left(-p_{1}-\right.$ $\left.p_{2}\right) A_{\mu}^{a}\left(p_{1}\right) A_{\nu}^{b}\left(p_{2}\right)|0\rangle$ is

$$
\begin{aligned}
R_{\lambda \mu \nu}^{a b}= & g^{2} T(\mathrm{R}) \delta^{a b} \int \frac{\mathrm{d}^{n} k}{(2 \pi)^{4}} \\
& \times \frac{\operatorname{Tr}\left[\gamma^{5} \gamma_{\lambda}\left(k+p_{1}\right) \gamma_{\mu} k \gamma_{\nu}\left(k-p_{2}\right)\right]-\operatorname{Tr}\left[\gamma^{5} \gamma_{\lambda}\left(k-p_{2}\right) \gamma_{\nu} k \gamma_{\mu}\left(k+p_{1}\right)\right]}{k^{2}\left(k+p_{1}\right)^{2}\left(k-p_{2}\right)^{2}} .
\end{aligned}
$$

Note that we have included trace terms corresponding to both fermion arrow directions in the loop, as appropriate for Dirac fermions. We have been careful to verify in every diagram that these two choices always give equal contributions. This means that the case of Majorana fermions can be extracted unambiguously by suitable choice of group theory factors.

Using the results of appendix $A$ it is straightforward to show that

$$
R_{\lambda_{\mu \nu}}\left(p_{1}, p_{2}\right)=\xi \cdot 2 \int_{0}^{1} \mathrm{~d} x \int_{0}^{1-x} \mathrm{~d} y K_{\lambda \mu \nu}
$$

where

$$
\begin{gathered}
K_{\lambda \mu \nu}=(n-4) J_{2} \operatorname{Tr}\left[\gamma^{5} \gamma_{\lambda} \gamma_{\mu} \gamma_{\nu}\left(p_{2}-p_{1}\right)\right]-Q^{2} J_{1} \operatorname{Tr}\left[\gamma^{5} \gamma_{\lambda} \gamma_{\mu} \gamma_{\nu}\left(\not p_{1}-p_{2}\right)\right] \\
+4 Q_{\nu} J_{1} \operatorname{Tr}\left[\gamma^{5} \gamma_{\lambda} p_{1} \gamma_{\mu} Q\right]-4 Q_{\mu} J_{1} \operatorname{Tr}\left[\gamma^{5} \gamma_{\lambda} Q \gamma_{\nu} p_{2}\right]+2 J_{1} \operatorname{Tr}\left[\gamma^{5} \gamma_{\lambda} p_{1} \gamma_{\mu} Q \gamma_{\nu} p_{2}\right] \\
\xi=\frac{g^{2}}{(2 \pi)^{4}} T(\mathrm{R}) \delta^{a h}
\end{gathered}
$$

(See appendix A for the definitions of $J_{1}, J_{2}$ and Q.)

Note that, since $J_{2}$ has a simple pole at $n=4$ while $J_{1}$ is finite, $R_{\lambda \mu \nu}$ is well-defined and finite at $n=4$. [Use of the identity (A.4) is crucial to the derivation of this result.1 
It follows easily from (3.7) that

$$
\begin{aligned}
\left(p_{1}+p_{2}\right)^{\lambda} R_{\lambda \mu \nu} & =\xi 4(n-4) J_{2} \operatorname{Tr}\left[\gamma^{5} \gamma_{\mu} \gamma_{\nu} p_{1} p_{2}\right] \\
& =2 \pi^{2} \xi \operatorname{Tr}\left[\gamma^{5} \gamma_{\mu} \gamma_{\nu} p_{1} \not p_{2}\right] .
\end{aligned}
$$

So

$$
A=2 T(\mathrm{R})
$$

For QED this is the usual anomaly. The diligent reader may care to verify from eq. (3.7) that

$$
p_{1}^{\mu} R_{\lambda \mu \nu}=p_{2}^{\nu} R_{\lambda \mu \nu}=0
$$

corresponding to vector current conservation.

The above calculation is an elegant demonstration of the power of dimensional regularization. In order to facilitate performance of the two-loop calculation, we modify the calculation as follows. Consider eq. (3.4). Differentiating both sides with respect to $p_{1}^{\alpha}$, and setting $p_{1}=-p_{2}=p$, we obtain

$$
R_{\alpha \mu \nu}^{a b}(p,-p)=\delta^{a b}\left[\frac{A g^{2}}{16 \pi^{2}}+\frac{B g^{4}}{\left(16 \pi^{2}\right)^{2}}\right] \cdot 4 \varepsilon_{\mu \nu \sigma \alpha} p^{\sigma}
$$

Thus to determine $A$ and $B$ it is sufficient to calculate $R_{\alpha \mu \nu}(p,-p)$. The one-loop calculation becomes

$$
\begin{aligned}
R_{\alpha \mu \nu}(p,-p)= & g^{2} T(\mathrm{R}) \delta^{a b} \int \frac{\mathrm{d}^{n} k}{(2 \pi)^{4}} \\
& \times \frac{\operatorname{Tr}\left[\gamma^{5} \gamma_{\alpha} k \gamma_{\mu}(k-p) \gamma_{\nu} k\right]-\operatorname{Tr}\left[\gamma^{5} \gamma_{\alpha} k \gamma_{\nu}(k-p) \gamma_{\mu} k\right]}{\left(k^{2}\right)^{2}(p-k)^{2}},
\end{aligned}
$$

whence (see appendix B)

$$
R_{\alpha \mu \nu}(p,-p)=\frac{g^{2} T(\mathrm{R})}{16 \pi^{2}} \delta^{a b}(n-4) I \operatorname{Tr}\left[\gamma^{5} \gamma_{\mu} \gamma_{\nu} \gamma_{\alpha} p\right]
$$

Taking the limit $n \rightarrow 4$ we find

$$
A=2 T(\mathrm{R})
$$


as before. This is a simpler method of obtaining $A$ (and in subsect. 4.1, B), for the elementary reason that integrals that depend on only one external momentum are easily done in closed form. A similar calculation gives $R_{\alpha \mu \nu}(p, 0)=0$ which (it is easy to see) corresponds to vector current conservation. We have verified that this result remains true at the two-loop level in QED.

Thus we have rederived the usual result for the one-loop VVA anomaly using dimensional regularization. It is clear [6] that the calculation is not modified at all if we use instead supersymmetric dimensional regularization (SDR). The only Dirac indices not contracted with momenta are $\mu$ and $\nu$, the indices on the external gluons. However, in SDR external gluons also bear $n$-dimensional indices. The easiest way to see this is to consider the gauge transformations

$$
\begin{aligned}
& \delta A_{\hat{\mu}}^{a}=g f^{a b c} A_{\hat{\mu}}^{b} \Lambda^{c}+\partial_{\hat{\mu}} \Lambda^{a}, \\
& \delta A_{\tilde{\mu}}^{a}=g f^{a b c} A_{\tilde{\mu}}^{b} \Lambda^{c}, \quad\left(\partial_{\tilde{\mu}} \Lambda^{a} \equiv 0\right) .
\end{aligned}
$$

Only the first $n$ components of $A_{\mu}$ behave as a vector under gauge transformations.

In sect. 4 we extend the calculation to the two-loop level and determine $B$. Because there are now graphs involving internal gluons CDR and SDR then give different results for individual graphs.

\section{The anomaly in next-to-leading order}

In this section, we describe the calculations of the constants $B$ and $C$ defined in eqs. (3.4) and (3.5). As explained earlier we have differentiated the diagrams with respect to $p_{1}^{\alpha}$ and set $p_{1}=-p_{2}=p$. For the sake of clarity, we will describe the calculation of $B$, i.e. of the matrix elements of the axial vector current at zero momentum transfer (the left-hand side) first. We then discuss the calculation of $C$, the matrix element of the operator $F \tilde{F}$ and its renormalization. We perform the calculation with conventional dimensional regularization and the supersymmetric dimensional regularization described in sect. 2.

For the convenience of the reader who wishes to reproduce our results, we will list the contributions of groups of diagrams separately in three tables. The notation for the standard one- and two-loop integrals used in writing the final answers is defined in appendix B. We have found it convenient to eliminate the $I^{2}$ terms and express everything in terms of $Z$ and the product $I J$. In this way an explicit factor of $(n-4)$ appears multiplying $I J$ in each term. All diagrams are proportional to the tensor structure $\operatorname{Tr} \gamma^{5} \gamma^{\alpha} \gamma^{\mu} \gamma^{\nu} p$, which we will omit to simplify already long formulae. We also set $p^{2}=1$ throughout, except in the final expressions for $B$ and $C$. Finally we use the Feynman gauge $(\alpha=1)$ throughout the calculation. 


\subsection{THE MATRIX ELEMENT OF $J_{\mu}^{5}(0)$}

The two-loop diagrams are shown in fig. 2. Before differentiation the divergence of the axial current introduces $\gamma^{5}\left(\not \phi_{1}+\phi_{2}\right)$ at the axial vertex. When we differentiate and set $p_{1}=-p_{2}=p$, a non-vanishing result is obtained only when differentiating the axial vertex. Consequently the calculation now reduces to the calculation of the matrix element of $J_{5}^{\mu}$ at zero momentum transfer.

For generality we perform the calculation with Dirac fermions and so there are two directions for the charge flow. The result for any diagram is invariant under reversing the charge flow, so each diagram except $2 \mathrm{i}$ and $2 \mathrm{j}$ can be multiplied by a factor of two and computed only for one arrow direction. For the diagrams $2 \mathrm{i}, \mathrm{j}$ reversing the fermion direction does not produce a topologically distinct diagram; consequently these diagrams are not multiplied by two. Only the counter-term diagrams $2 m-r$ require an explanation. They represent the subtraction of the pole term of the appropriate self-energy or vertex insertion.

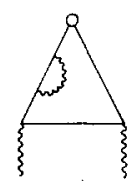

(a)

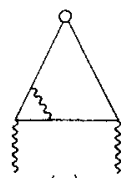

(e)

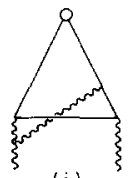

(i)

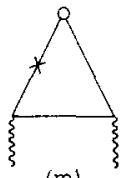

(m)

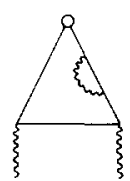

(b)

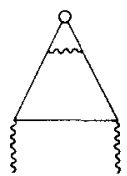

(f)

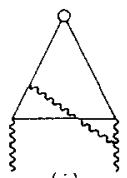

(j)

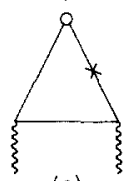

(n)

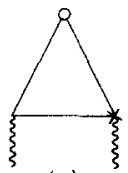

(a)

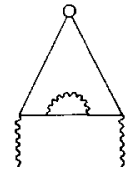

(c)

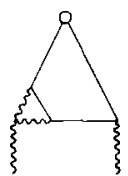

(g)

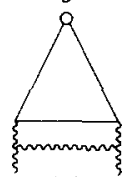

(k)

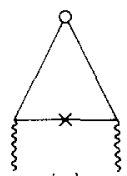

(o)

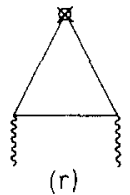

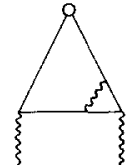

(d)

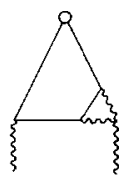

(h)

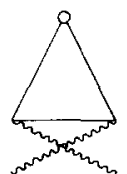

(l)

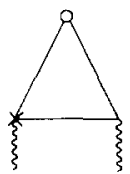

(p)

Fig. 2. The second-order contribution to the axial anomaly. For Dirac fermions there are two directions of charge flow. Flipping the charge direction does not yield a topologically distinct diagram for (i) and (j). A cross represents the insertion of the appropriate $\mathrm{O}\left(g^{2}\right)$ counter-term. 
The only subtlety arises in the calculation of fig. $2 r$. It is necessary to ensure that the axial current is correctly normalized, and this determines the subtraction constant at the top vertex. We do this by imposing the identity

$$
\Gamma_{\mu}^{5}=\gamma^{5} \Gamma_{\mu}
$$

relating the renormalized axial and vector two-fermion Green functions. In the calculation of $\Gamma_{\mu}$ we subtract only the pole term in $\varepsilon$; it therefore follows, if we assume an anticommuting $\gamma^{5}$, that we must subtract only the pole term in $\Gamma_{\mu}^{5}$ too. Conversely, if we adopt the $\gamma^{5}$ prescription of eq. (2.5), then there are additional finite pieces which must also be subtracted. Thus, in this case, the identity (4.1) is true only for the renormalized (and not the unrenormalized) Green functions. As we shall see it is this procedure which leads to the $A B$ theorem. Thus we obtain for fig. $2 \mathrm{r}$

$$
[-2 I+\chi] C_{2}(\mathrm{R}) T(\mathrm{R}),
$$

where the first term arises from the pole term in the subtraction, and $\chi$ is given by

$$
\chi_{\mathrm{CDR}}=\chi_{\mathrm{SDR}}=0,
$$

for an anticommuting $\gamma^{5}$, and

$$
\begin{aligned}
& \chi_{\mathrm{CDR}}=-8, \\
& \chi_{\mathrm{SDR}}=-4,
\end{aligned}
$$

for the $\gamma^{5}$ of eq. (2.5). The different results of eq. (4.4) and (4.5) arise because in the former case the $\gamma^{5}$ must commute past two $n$-dimensional $\gamma$ matrices, while in the former one of them is $n$-dimensional and the other 4-dimensional. One might be concerned that in the SDR case there is a contradiction between eq. (2.5) which requires $n>4$ and dimensional reduction, which requires $n<4$. However, the properties of $\gamma^{5}$ required are

$$
\begin{aligned}
\gamma^{\hat{\mu}} \gamma^{5} \gamma^{\hat{\mu}} & =(n-8) \gamma^{5}, \\
\gamma^{\hat{\mu}}\left[\gamma^{5}, \gamma^{\hat{}}\right] \gamma^{\hat{\mu}} & =(6-n)\left[\gamma^{5}, \gamma^{\hat{\nu}}\right] .
\end{aligned}
$$

These relations can presumably be extended to $n<4$, formally at least. Of course, it is not clear that this procedure can be made consistent with supersymmetry, which serves once again to underline the unsatisfactory state of the SDR procedure. All the integrals necessary to evaluate the diagrams have been tabulated in appendix B. An integration by parts is needed before the non-planar diagrams $2 i, j$ are integrable 
with the integrals of appendix B. The results of the diagrams are summarized in table 1 for CDR and table 2 for SDR. (Diagram 21 vanishes identically after the differentiation has been performed.) Note that the $Z$ contribution to all diagrams except $\mathrm{g}+\mathrm{h}$ and $\mathrm{i}+\mathrm{j}$ is proportional to $(n-4)$. The $Z$ term of the sum $\mathrm{g}+\mathrm{h}+\mathrm{i}+\mathrm{j}$ also contains an $(n-4)$ factor as can be easily verified. Since the expression for $Z$ is finite as $n=4$, namely $Z=6 \zeta(3) \pi^{4}$, the total $Z$ contribution will vanish as $n \rightarrow 4$. Note also that the $I J$ contributions have simple poles for all diagrams. The pole terms cancel in the sum as they should.

Taking the $n \rightarrow 4$ limit, the sum of all diagrams becomes

$$
\begin{aligned}
& B_{\mathrm{CDR}}=\left(8+\chi_{\mathrm{CDR}}\right) C_{2}(\mathrm{R}) T(\mathrm{R})+C_{2}(\mathrm{G}) T(\mathrm{R})(12-4 \gamma), \\
& B_{\mathrm{SDR}}=\left(4+\chi_{\mathrm{SDR}}\right) C_{2}(\mathrm{R}) T(\mathrm{R})+C_{2}(\mathrm{G}) T(\mathrm{R})(12-4 \gamma),
\end{aligned}
$$

TABLE 1

Contribution of diagrams shown in fig. 2 in CDR for general $n$

\begin{tabular}{lll}
\hline Diagram & \multicolumn{1}{c}{ Group factor } & Result $/ \operatorname{Tr}\left(\gamma^{5} \gamma^{\alpha} \gamma^{\mu} \gamma^{\nu} p\right) \cdot \delta^{a b}\left(g^{4} /\left(16 \pi^{2}\right)^{2}\right)$ \\
$\mathrm{a}+\mathrm{b}+\mathrm{c}$ & $C_{2}(\mathrm{R}) T(\mathrm{R})$ & $\frac{2(n-4)(n-2)\left(-n^{2}+12 n-24\right)}{(6-n)(3 n-8)} I J$ \\
$\mathrm{~d}+\mathrm{e}$ & $T(\mathrm{R})\left[C_{2}(\mathrm{R})-\frac{1}{2} C_{2}(\mathrm{G})\right]$ & $\frac{(n-4)(n-2)\left(n^{2}-7 n+15\right)}{(n-1)(n-3)} Z$ \\
& & $\frac{2(n-2)(n-4)\left(-7 n^{3}+69 n^{2}-227 n+246\right)}{(n-1)(n-3)(3 n-8)} I J$ \\
$\mathrm{f}$ & $C_{2}(\mathrm{R}) T(\mathrm{R})$ & $\frac{2(n-4)(n-6)(n-2)}{3(n-8)} I J$ \\
$\mathrm{~g}+\mathrm{h}$ & $\frac{1}{2} T(\mathrm{R}) C_{2}(\mathrm{G})$ & $\frac{-(n-2)^{2}}{(n-1)} Z+\frac{2(n-4)\left(-5 n^{3}+28 n^{2}-56 n+48\right)}{(n-1)(6-n)(3 n-8)} I J$ \\
$\mathrm{i}+\mathrm{j}$ & $\frac{1}{2} T(\mathrm{R}) C_{2}(\mathrm{G})$ & $\frac{2\left(-2 n^{2}+17 n-34\right)}{(n-1)(n-3)} Z$ \\
& & $+\frac{4(n-4)\left(8 n^{4}-108 n^{3}+561 n^{2}-1304 n+1128\right)}{(n-1)(n-3)(6-n)(3 n-8)} I J$ \\
$\mathrm{k}$ & $T(\mathrm{R}) C_{2}(\mathrm{G})$ & $4(n-4) \frac{\left(4 n^{2}-33 n+58\right)}{(3 n-8)(6-n)} I J$ \\
$\mathrm{~m}+\mathrm{n}+\mathrm{o}$ & $C_{2}(\mathrm{R}) T(\mathrm{R})$ & $6 I$ \\
$\mathrm{p}+\mathrm{q}$ & $C_{2}(\mathrm{R}) T(\mathrm{R})$ & $-4 I$ \\
$\mathrm{r}$ & $+C_{2}(\mathrm{G}) T(\mathrm{R})$ & $-2 I+\chi$ \\
\hline
\end{tabular}

We have eliminated $I^{2}$ using identity (B.4) Diagram 1 vanishes identically. The answer for each is the product of the group factor and the last column. 
TABLE 2

Table 1 with $\mathrm{CDR} \rightarrow \mathrm{SDR}$

\begin{tabular}{|c|c|c|}
\hline Diagram & Group factor & Result $/ \operatorname{Tr}\left(\gamma^{5} \gamma^{\alpha} \gamma^{\mu} \gamma^{\nu} p\right) \cdot \delta^{a b}\left(g^{4} /\left(16 \pi^{2}\right)^{2}\right)$ \\
\hline$a+b+c$ & $C_{2}(\mathrm{R}) T(\mathrm{R})$ & $\frac{4(n-4)\left(-n^{2}+12 n-24\right)}{(6-n)(3 n-8)} I J$ \\
\hline $\mathrm{d}+\mathrm{e}$ & $T(\mathrm{R})\left[C_{2}(\mathrm{R})-\frac{1}{2} C_{2}(\mathrm{G})\right]$ & $\frac{2(n-4)\left(n^{2}-5 n+7\right)}{(n-1)(n-3)} Z+\frac{4(n-4)\left(-3 n^{3}+27 n^{2}-83 n+86\right)}{(n-1)(n-3)(3 n-8)} I J$ \\
\hline f & $C_{2}(\mathrm{R}) T(\mathrm{R})$ & $\frac{4(n-4)(2-n)}{(3 n-8)} I J$ \\
\hline$g+h$ & $\frac{1}{2} T(\mathrm{R}) C_{2}(\mathrm{G})$ & $\frac{\left(-4 n^{3}+38 n^{2}-128 n+152\right)}{2(n-1)(n-3)} Z$ \\
\hline$i+j$ & $\frac{1}{2} T(\mathrm{R}) C_{2}(\mathrm{G})$ & $\begin{array}{l}+\frac{2(n-4)\left(-12 n^{4}+155 n^{3}-790 n^{2}+1844 n-1632\right)}{(n-1)(n-3)(6-n)(3 n-8)} I J \\
\frac{\left(-3 n^{2}+26 n-52\right)}{(n-1)(n-3)} Z \\
+\frac{2(n-4)\left(14 n^{4}-183 n^{3}+924 n^{2}-2096 n+1776\right)}{(n-1)(n-3)(6-n)(3 n-8)} I J\end{array}$ \\
\hline $\mathrm{k}$ & $T(\mathrm{R}) C_{2}(\mathrm{G})$ & $\frac{4(n-4)\left(4 n^{2}-33 n+58\right)}{(6-n)(3 n-8)} I J$ \\
\hline $\mathrm{m}+\mathrm{n}+\mathrm{o}$ & $C_{2}(\mathrm{R}) T(\mathrm{R})$ & $6 I$ \\
\hline$p+q$ & $\begin{array}{l}C_{2}(\mathrm{R}) T(\mathrm{R}) \\
+C_{2}(\mathrm{G}) T(\mathrm{R})\end{array}$ & $-4 I$ \\
\hline $\mathbf{r}$ & $C_{2}(\mathrm{R}) T(\mathrm{R})$ & $-2 I+\chi$ \\
\hline
\end{tabular}

where $\gamma=\gamma_{\mathrm{E}}+\ln \left(p^{2} / \mu^{2}\right), \gamma_{\mathrm{E}}$ being Euler's constant and $\mu^{2}$ the renormalization scale. The group theory factors are according to the usual convention:

$$
\begin{gathered}
T(\mathrm{R}) \delta^{a b}=\operatorname{Tr} R^{a} R^{b}, \\
C_{2}(\mathrm{G}) \delta^{a b}=f^{a c d} f^{b c d}, \\
C_{2}(\mathrm{R}) I=R^{a} R^{a} .
\end{gathered}
$$

\subsection{THE MATRIX ELEMENT OF $g^{2} F \tilde{F}$}

We must calculate the one-loop diagrams shown in fig. 3. The Feynman rules for the $F \tilde{F}$ vertex are easily obtained by expanding the operator, and are shown in fig. 4 . To compute the diagrams we first write down the exact expression with momenta $p_{1}$ and $p_{2}$ flowing into the external gluon lines. We then differentiate with respect to $p_{1}^{\alpha}$ and set $p_{1}=-p_{2}=p$. Because the three-gluon vertex in fig. 4 is proportional to the 


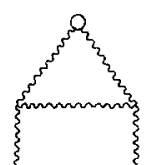

(a)

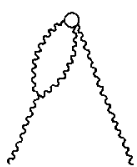

(b)

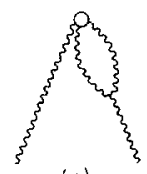

(c)

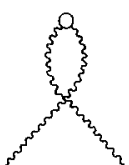

(d)

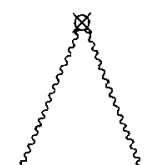

(e)

Fig. 3. The correction to the matrix elements for $F \tilde{F}$ in second order. The circle denotes the insertion of the operator. Diagram (e) represents the $\mathrm{O}\left(\mathrm{g}^{2}\right)$ renormalization of the operator.

total momentum flowing into the vertex, we only need to differentiate the $F \tilde{F}$ vertex for figs. $3 b, c$. It is easy to verify that for fig. 3a we only need to differentiate the ordinary three-gluon vertex. Fig. $3 d$ vanishes identically after differentiation since no momentum flows in the loop. Hence we never differentiate a propagator inside the graph; consequently, we do not generate any infrared divergences. When calculating the diagrams in either dimensional method, we must continue to $n$ dimensions. The Feynman rules of fig. 4 contain explicitly the $\varepsilon$ tensor; so we must define what we mean by these rules in $n$ dimensions. Actually there is a natural definition namely we identify the $\varepsilon_{\mu \nu \rho \sigma}$ tensor with $(1 / 4 i) \operatorname{Tr}\left(\gamma^{5} \gamma_{\mu} \gamma_{\nu} \gamma_{\rho} \gamma_{\sigma}\right)$ which we can easily continue to $n$ dimensions. The only property of the tensor which is needed in the calculation is the complete antisymmetry in the four Lorentz indices, which is satisfied by the trace tensor if $\operatorname{Tr} \gamma^{5} \gamma^{\mu} \gamma^{\nu}=0$ in $n$ dimensions which we have assumed.

Fig. 3e provides the renormalization of the operator $g^{2} F \tilde{F}$. The bare operator $g_{0}^{2} F_{0} \tilde{F}_{0}$ is renormalized by the usual rescaling: $g_{0}=Z_{\mathrm{g}} g=Z_{1} Z_{A}^{-3 / 2} \mathrm{~g}, A_{0}^{\mu}=Z_{A}^{1 / 2} A^{\mu}$. If we carry out this rescaling, we find to order $g^{4}$

$$
g_{0}^{2} F_{0} \tilde{F}_{0}=g^{2} F \tilde{F}-g^{2}\left(1-Z_{1}^{2} Z_{A}^{-2}\right) F \tilde{F}+\cdots
$$

The second term in this equation is the counter-term which we need to renormalize the bare operator $g_{0}^{2} F_{0} \tilde{F}_{0}$. Given the standard formulae for the renormalization

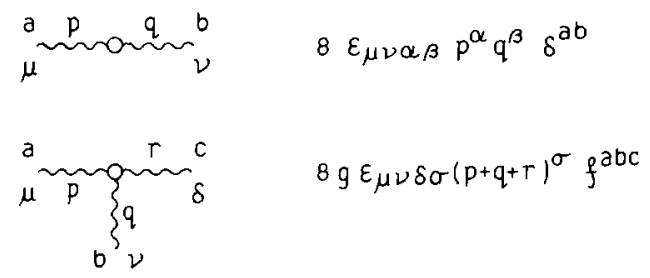

Fig. 4. The Feynman rules for the operator $F \tilde{F}$. 
constants (in the Feynman gauge),

$$
\begin{aligned}
& Z_{A}=1+\frac{g^{2}}{16 \pi^{2}}\left(\frac{10}{3} C_{2}(\mathrm{G})-\frac{8}{3} T(\mathrm{R})\right) \frac{1}{4-n} \\
& Z_{1}=1+\frac{g^{2}}{16 \pi^{2}}\left(\frac{4}{3} C_{2}(\mathrm{G})-\frac{8}{3} T(\mathrm{R})\right) \frac{1}{4-n}
\end{aligned}
$$

we find

$$
g^{2}\left(1-Z_{1}^{2} Z_{A}^{-2}\right)=-\frac{4 g^{4}}{16 \pi^{2}} \frac{C_{2}(\mathrm{G})}{4-n}
$$

Note that the fermion contributions to $Z_{1}$ and $Z_{A}$ cancel in the renormalization of $g^{2} F \tilde{F}$.

This rescaling of $g^{2} F \tilde{F}$ is sufficient to render the two-gluon matrix element of this operator finite: there is no anomalous dimension. We are now ready to give the result of the computation. It is easy to verify that the result is identical for CDR and SDR. The contribution for each graph is summarized in table 3 . The total contribution is finite as $n \rightarrow 4$. We easily find [taking into account the coefficient 2 factored out in eq. (3.5)]

$$
C=(6-2 \gamma) C_{2}(\mathrm{G})
$$

If we now define $r$ by the relation

$$
\partial \cdot J^{5}=r F \tilde{F}
$$

and substitute the values of $A, B$ and $C$ from eqs. (3.8), (4.7) and (4.11) in eqs. (3.4)

TABLE 3

Contributions of diagrams in fig. 3

\begin{tabular}{cc}
\hline Diagram & Result $/\left[\left(\operatorname{Tr} \gamma^{5} \gamma^{\alpha} \gamma^{\mu} \gamma^{\nu} p\right) \cdot \delta^{a h} g^{4} / 16 \pi^{2}\right]$ \\
\hline $\mathrm{a}+\mathrm{c}$ & $-2(n-9) I C_{2}(\mathrm{G})$ \\
$\mathrm{b}+\mathrm{c}$ & $-6 I C_{2}(\mathrm{G})$ \\
$\mathrm{d}$ & 0 \\
$\mathrm{e}$ & $+\frac{8 C_{2}(G)}{n-4}$ \\
\hline
\end{tabular}

CDR and SDR give identical results. 
and (3.5), we find

$$
\begin{aligned}
& r_{\mathrm{CDR}}=\frac{T(\mathrm{R}) g^{2}}{16 \pi^{2}}+\frac{C_{2}(\mathrm{R}) T(\mathrm{R}) g^{4}}{\left(16 \pi^{2}\right)^{2}}\left(4+\frac{1}{2} \chi_{\mathrm{CDR}}\right), \\
& r_{\mathrm{SDR}}=\frac{T(\mathrm{R}) g^{2}}{16 \pi^{2}}+\frac{C_{2}(\mathrm{R}) T(\mathrm{R}) g^{4}}{\left(16 \pi^{2}\right)^{2}}\left(2+\frac{1}{2} \chi_{\mathrm{SDR}}\right) .
\end{aligned}
$$

Note that in both cases the dependence on $C_{2}(\mathrm{G})$ cancels.

From eqs. (4.13) and (4.14) it is at once apparent that the $\mathrm{AB}$ theorem is valid for both CDR and SDR if we use the values of $\chi$ from eqs. (4.4) and (4.5). So dimensional regularization in either form correctly reproduces the $\mathrm{AB}$ theorem to this order providing we are careful to normalize the current correctly by imposing the identity (4.1), and use consistently the 't Hooft-Veltman $\gamma^{5}$.

Supersymmetric Yang-Mills theory corresponds to the special case $C_{2}(\mathrm{R})=C_{2}(\mathrm{G})$ $=2 T(\mathrm{R}) . \mathrm{Eq} .(4.14)$ then yields

$$
r_{\mathrm{SDR}}=\frac{1}{2} \frac{C_{2}(\mathrm{G})}{16 \pi^{2}} g^{2}+\frac{C_{2}(\mathrm{G})^{2}}{\left(16 \pi^{2}\right)^{2}} g^{4}\left(1+\frac{1}{4} \chi_{\mathrm{SDR}}\right)
$$

We now require the $\beta$ function through two loops. This was calculated using CDR in ref. [27], and it was subsequently verified that SDR gives the same results (Capper et al., ref. [18]), namely

$$
\beta(g)=-3 \frac{C_{2}(\mathrm{G})}{16 \pi^{2}} g^{3}-6\left[\frac{C_{2}(\mathrm{G})}{16 \pi^{2}}\right]^{2} g^{5}
$$

So we see that, in accordance with eq. (3.1),

$$
r_{\mathrm{SDR}}=-\frac{1}{6} \beta(g)
$$

if and only if $\chi_{\mathrm{SDR}}=0$.

Thus we obtain the conjectured supersymmetric result by using an anticommuting $\gamma^{5}$ in the calculation of $\Gamma_{\mu}^{5}$. While this is an intriguing result it should be emphasized that its significance is unclear. Although the other graphs of fig. 2 were calculated without assuming any property of $\gamma^{5}$ other than $\operatorname{Tr} \gamma^{5} \gamma_{\alpha} \gamma_{\beta}=0$ the fact remains that strictly speaking a fully anticommuting $\gamma^{5}$ gives zero for each graph because of the identity eq. (2.4), while the $\gamma^{5}$ of eq. (2.5) would reproduce our results. Therefore one could argue that the result $r \sim \beta(g)$ is obtained only at the expense of the chiral identity, eq. (4.1). 


\section{Conclusions}

We have verified that, using conventional dimensional regularization and the non-anticommuting $\gamma^{5}$ of 't Hooft and Veltman, the Adler-Bardeen theorem is valid through two-loops in both abelian and non-abelian gauge theories. With supersymmetric dimensional regularization the same result is obtained, although in the SDR case use of a non-anticommuting $\gamma^{5}$ is somewhat paradoxical since SDR requires $n<4$.

If we use an anticommuting $\gamma^{5}$ in the calculation of $\Gamma_{\mu}^{5}$ we obtain apparent violation of the $\mathrm{AB}$ theorem even in the case of QED. As already emphasized, this arises from the fact that the normalization of the axial current is now inconsistent with eq. (4.1). It is nevertheless intriguing that in the case of supersymmetric Yang-Mills theory, however, this method (with SDR) gives $\partial \cdot J^{5} \sim \beta(g) F \tilde{F}$ in accordance with expectations based on the supposed supermultiplet structure of the currents. Since it is not clear what restriction supersymmetry places on the axial current normalization, the question of whether the Adler-Bardeen theorem is valid in supersymmetric theories remains undecided.

It is amusing to note that in the cases of $N=2$ and $N=4$ supersymmetric Yang-Mills theory there is, in any case, no apparent conflict between supersymmetry and the Adler-Bardeen theorem (at least to this order). For general $N, \beta(g)$ is given by $[28]$

$$
\beta(g)=(N-4) \frac{C_{2}(\mathrm{G})}{16 \pi^{2}} g^{3}-2(N-4)(N-2)\left[\frac{C_{2}(\mathrm{G})}{16 \pi^{2}}\right]^{2} g^{5},
$$

so that the two-loop contribution vanishes for both $N=4$ and $N=2$. In the case $N=4$ it is generally believed (and verified through $\mathrm{O}\left(g^{7}\right)$ ) [29] that $\beta(g)$ vanishes to all orders. In the case $N=2$ it has been conjectured that the one-loop result is exact [30]. In both cases (assuming the truth of the conjectures) there would be no conflict with the AB theorem. Thus it is $N=1$ SSYM that appears "anomalous" (sic) from the above point of view.

One of the rules of the game of constructing renormalized gauge theories of unified interactions is that gauged axial currents should be kept anomaly free. This is commonly achieved by choosing particle representations such that the anomaly cancels at one loop and appealing to the $\mathrm{AB}$ theorem to extend this result to all orders. In the model we considered the axial current was not gauged; however, it is clear that the supermultiplet structure of the gauged axial currents and the question of anomaly cancellation in supersymmetric unified models requires careful examination. Must one impose an infinite set of anomaly constraints? Or is it, in fact, the case that a regulator exists such that the one-loop result is exact for all three anomalies? A final resolution of these questions requires a superfield formulation of the anomalies and a two-loop calculation with a manifestly supersymmetric regulator, free of the ambiguities discussed in sect. 2. Work in this direction is in progress. 
We thank our colleagues at The University of Michigan (especially M. Einhorn) and Eric Braaten for discussions, and the Institute of Theoretical Physics, Santa Barbara (where this work was begun) for its hospitality. One of us (DRTJ) thanks seminar audiences at Stony Brook and Harvard for stimulating criticism. This work was supported in part by the US Department of Energy.

\section{Appendix A}

In this appendix we derive an identity the use of which is crucial to the derivation of eq. (3.5), and hence to the demonstration of the finiteness of the VVA triangle graph. In fact the derivation was given by Capper [25]; we reproduce it here for convenience.

We write

$$
\begin{aligned}
& \int \frac{\mathrm{d}^{n} k}{k^{2}\left(k+p_{1}\right)^{2}\left(k-p_{2}\right)^{2}}=2 \int_{0}^{1} \mathrm{~d} x \int_{0}^{1-x} \mathrm{~d} y J_{1}, \\
& \int \frac{\mathrm{d}^{n} k k_{\mu} k_{\nu}}{k^{2}\left(k+p_{1}\right)^{2}\left(k-p_{2}\right)^{2}}=2 \int_{0}^{1} \mathrm{~d} x \int_{0}^{1-x} \mathrm{~d} y\left(J_{2} \delta_{\mu \nu}+J_{1} Q_{\mu} Q_{\nu}\right),
\end{aligned}
$$

where

$$
\begin{aligned}
& Q=p_{1} y-p_{2} x \\
& J_{1}=\frac{1}{2} \pi^{n / 2} \Gamma\left(3-\frac{1}{2} n\right) D^{(n / 2)-3}, \\
& J_{2}=\frac{D J_{1}}{4-n},
\end{aligned}
$$

and

$$
D=x(1-x) p_{2}^{2}+y(1-y) p_{1}^{2}+2 x y p_{1} \cdot p_{2} .
$$

Consider the identity

$$
\int \frac{\left(2 k^{\alpha}+p_{1}^{\alpha}-p_{2}^{\alpha}\right) k^{2} \mathrm{~d}^{n} k}{k^{2}\left(k+p_{1}\right)^{2}\left(k-p_{2}\right)^{2}}=0
$$

(easily shown by shifting $k \rightarrow-k+p_{1}-p_{2}$ ). In terms of $J_{1}, J_{2}$ this identity becomes

$$
\int_{0}^{1} \mathrm{~d} x \int_{0}^{1-x} \mathrm{~d} y(n+2) J_{2} Q^{\alpha}\left\{-\frac{1}{2}\left(p_{1}^{\alpha}-p_{2}^{\alpha}-2 Q^{\alpha}\right) Q^{2} J_{1}-\frac{1}{2} n\left(p_{1}^{\alpha}-p_{2}^{\alpha}\right) J_{2}\right\}=0 .
$$


Eq. (A.4) is the identity required for the derivation of eq. (3.5). An analogous identity can be derived for the case of massive fermions [25].

\section{Appendix B}

\section{THE TWO-LOOP INTEGRALS}

For our calculation we require a number of two-loop integrals dependent on one external momentum. We find it convenient to express all these integrals in terms of three basic quantities $(I, J, Z)$ which are defined as follows ${ }^{\star}$ :

$$
\begin{array}{r}
\int \frac{\mathrm{d}^{n} k}{k^{2}(k+p)^{2}}=I, \\
\int \frac{\mathrm{d}^{n} k}{\left(k^{2}\right)^{3-n / 2}(k+p)^{2}}=J, \\
\int \frac{\mathrm{d}^{n} k \mathrm{~d}^{n} q}{q^{2} k^{2}(k+p)^{2}(q+p)^{2}(k-q)^{2}}=Z .
\end{array}
$$

In fact there exists a remarkably simple identity relating $I, J, Z$ :

$$
(n-4) Z=(6 n-20) I J-(2 n-6) I^{2}
$$

This identity is derived in ref. [31], and was rediscovered independently (and less directly) by the present authors. It represents a considerable simplification in the calculation of $Z$ over previous methods. It is easy to verify that the known result for $Z$, viz.

$$
Z=6 \zeta(3) \pi^{4}+\mathrm{O}(n-4)
$$

follows from eq. (B.4).

The two-loop integrals which we encounter are expressed in terms of $I, J$, and $Z$ by a process we illustrate by means of an example:

Consider the integral

$$
F^{\mu \nu}=\int \frac{\mathrm{d}^{n} k \mathrm{~d}^{n} q k^{\mu} k^{\nu}}{k^{2} q^{2}(p+q)^{2}(p+k)^{2}(k-q)^{2}} .
$$

* Throughout this paper (except where explicitly stated) we set $p^{2}=1$. 
Step I. Write the integral in terms of Lorentz invariant amplitudes $(a, b, \ldots)$, etc:

$$
F^{\mu v}=a \delta^{\mu v} p^{2}+b p^{\mu} p^{v}
$$

Step 1I. By contractions with Kronecker $\delta$ 's or $p^{\mu}, p^{v}$ derive a system of linear equations for $(a, b \ldots)$ :

$$
\begin{aligned}
& n a+b=I J, \\
& a+b=\frac{n-2}{2(3 n-8)} I J+\frac{1}{4} Z .
\end{aligned}
$$

Step III. Solve the resulting equations:

$$
\begin{aligned}
& a=\frac{5 n-14}{2(n-1)(3 n-8)} I J-\frac{1}{4(n-1)} Z . \\
& b=\frac{(n-4)^{2}}{2(n-1)(3 n-8)} I J+\frac{n}{4(n-1)} Z .
\end{aligned}
$$

It was this process applied to the integral

$$
\int \frac{\mathrm{d}^{n} k \mathrm{~d}^{n} q k^{\mu} k^{v} q^{\alpha} q^{\beta}}{k^{4} q^{2}(k+p)^{2}(q+p)^{2}(k-q)^{2}}
$$

that led to the identity (B.4). Step II gave more equations than invariants, and (B.3) was necessary for consistency of the equations.

We conclude this appendix by listing the results for some integrals that were used in the calculations of sect. 4 . We define

$$
\frac{1}{D} \equiv \frac{\mathrm{d}^{n} k \mathrm{~d}^{n} q}{k^{2} q^{2}(k+p)^{2}(q+p)^{2}(k-q)^{2}} .
$$

Thus

$$
Z=\int \frac{1}{D}, \quad \text { etc }
$$

We find

$$
\begin{aligned}
\int \frac{k^{\mu} k^{\nu} q^{\lambda}}{D}= & p^{\mu} p^{\nu} p^{\lambda}\left[\frac{n^{2}+12 n-48}{4(n-1)(3 n-8)} I J-\frac{(n+2)}{8(n-1)} Z-\frac{1}{2(n-1)} I^{2}\right] \\
& +p^{\lambda} \delta^{\mu \nu}\left[\frac{14-5 n}{4(n-1)(3 n-8)} I J+\frac{1}{8(n-1)} Z\right]
\end{aligned}
$$




$$
\begin{aligned}
& +\left(p^{\mu} \delta^{\nu \lambda}+p^{\nu} \delta^{\mu \lambda}\right)\left[\frac{20-7 n}{4(n-1)(3 n-8)} I J\right. \\
& \left.+\frac{1}{8(n-1)} Z+\frac{1}{4(n-1)} I^{2}\right] \\
& \int \frac{k^{\mu} k^{\nu} q^{\lambda}}{k^{2} D}=p^{\mu} p^{\nu} p^{\lambda} \frac{(n-5)}{4(n-1)}\left[\frac{-\left(n^{2}+2 n-16\right)}{3 n-8} I J+\frac{1}{2} n Z+I^{2}\right] \\
& +\delta^{\mu \nu} p^{\lambda} \frac{1}{4(n-1)}\left[\frac{-2\left(4 n^{2}-23 n+32\right)}{3 n-8} I J-Z+(n-3) I^{2}\right] \\
& +\left(\delta^{\mu \lambda} p^{\nu}+\delta^{\nu \lambda} p^{\mu}\right) \frac{(n-4)}{4(n-1)}\left[\frac{7 n-20}{3 n-8} I J-\frac{1}{2} Z-I^{2}\right] \\
& \int \frac{k^{\mu} k^{\nu} k^{\lambda}}{k^{2} D}=\frac{5(n-3)(n-4)^{2}}{4(n-1)(n-6)(3 n-8)}\left[\delta^{\mu \nu} p^{\lambda}+\delta^{\mu \lambda} p^{\nu}+\delta^{\nu \lambda} p^{\mu}-(n+2) p^{\mu} p^{\nu} p^{\lambda}\right] I J \\
& +\frac{1}{8(n-1)}\left[(3-n)\left(\delta^{\mu \nu} p^{\lambda}+\delta^{\mu \lambda} p^{\nu}+\delta^{\nu \lambda} p^{\mu}\right)\right. \\
& \left.+\left(n^{2}-5 n-2\right) p^{\mu} p^{\nu} p^{\lambda}\right] Z \\
& \int \frac{k^{\mu} k^{\nu} k^{\lambda} q^{\alpha}}{k^{2} D}=A p^{\mu} p^{\nu} p^{\lambda} p^{\alpha}+B\left[\delta^{\mu \nu} p^{\lambda} p^{\alpha}+\delta^{\mu \lambda} p^{\nu} p^{\alpha}+\delta^{\nu \lambda} p^{\mu} p^{\alpha}\right] \\
& +C\left[\delta^{\lambda \alpha} p^{\mu} p^{\nu}+\delta^{\nu \alpha} p^{\mu} p^{\lambda}+\delta^{\mu \alpha} p^{\nu} p^{\lambda}\right]+D\left[\delta^{\mu \nu} \delta^{\lambda \alpha}+\delta^{\mu \lambda} \delta^{\nu \alpha}+\delta^{\nu \lambda} \delta^{\mu \alpha}\right],
\end{aligned}
$$

where

$$
\begin{aligned}
A= & \frac{11 n^{5}-43 n^{4}-516 n^{3}+2752 n^{2}-824 n-7680}{24\left(n^{2}-1\right)(n-6)(3 n-8)} I J \\
& -\frac{\left(n^{3}-n^{2}-16 n-20\right)}{16\left(n^{2}-1\right)} Z-\frac{3\left(n^{2}-3 n-6\right)}{8\left(n^{2}-1\right)} I^{2}, \\
B= & \frac{-\left(11 n^{4}-99 n^{3}+168 n^{2}+436 n-1056\right)}{24\left(n^{2}-1\right)(n-6)(3 n-8)} I J+\frac{\left(n^{2}-n-4\right)}{16\left(n^{2}-1\right)} Z+\frac{1}{8(n+1)} I^{2}, \\
C= & \frac{-\left(23 n^{4}-261 n^{3}+798 n^{2}+88 n-2208\right)}{24\left(n^{2}-1\right)(n-6)(3 n-8)} I J+\frac{\left(n^{2}-3 n-6\right)}{16\left(n^{2}-1\right)}\left(Z+2 I^{2}\right),
\end{aligned}
$$




$$
\begin{gathered}
D=\frac{23 n^{2}-200 n+384}{24(n+1)(n-6)(3 n-8)} I J-\frac{1}{16(n+1)}\left(Z+2 I^{2}\right) \\
\int \frac{k^{\alpha} k^{\beta} q^{\mu} q^{\nu}}{k^{2} D}= \\
=\bar{A} p^{\mu} p^{\nu} p^{\alpha} p^{\beta}+\bar{B} \delta^{\mu \nu} p^{\alpha} p^{\beta}+\bar{C} \delta^{\alpha \beta} p^{\mu} p^{\nu} \\
+\bar{D}\left[p^{\nu} p^{\alpha} \delta^{\mu \beta}+p^{\mu} p^{\beta} \delta^{\nu \alpha}+p^{\nu} p^{\beta} \delta^{\mu \alpha}+p^{\mu} p^{\alpha} \delta^{\nu \beta}\right] \\
+\bar{E} \delta^{\mu \nu} \delta^{\alpha \beta}+\bar{F}\left[\delta^{\mu \beta} \delta^{\nu \alpha}+\delta^{\nu \beta} \delta^{\mu \alpha}\right]
\end{gathered}
$$

where

$$
\begin{aligned}
\bar{A}= & \frac{-\left(5 n^{5}-55 n^{4}+378 n^{3}-1676 n^{2}+2488 n+960\right)}{24\left(n^{2}-1\right)(n-6)(3 n-8)} I J \\
& \frac{-n\left(n^{2}-3 n-10\right)}{16\left(n^{2}-1\right)} Z-\frac{\left(n^{2}-7 n-2\right)}{8\left(n^{2}-1\right)} I^{2} \\
\bar{B}= & \frac{-\left(19 n^{4}-255 n^{3}+1008 n^{2}-892 n-960\right)}{24\left(n^{2}-1\right)(n-6)(3 n-8)} I J+\frac{\left(n^{2}-5 n-4\right)}{16\left(n^{2}-1\right)} Z-\frac{1}{8(n+1)} I^{2} \\
\bar{C}= & \frac{32 n^{4}-318 n^{3}+756 n^{2}+400 n-1920}{24\left(n^{2}-1\right)(n-6)(3 n-8)} I J+\frac{(n+2)}{8\left(n^{2}-1\right)} Z-\frac{\left(n^{2}-n-4\right)}{8\left(n^{2}-1\right)} I^{2}, \\
\bar{D}= & \frac{8 n^{4}-60 n^{3}+18 n^{2}+532 n-768}{24\left(n^{2}-1\right)(n-6)(3 n-8)} I J+\frac{1}{8\left(n^{2}-1\right)} Z-\frac{1}{8(n+1)} I^{2}, \\
\bar{E}= & \frac{-\left(8 n^{3}-106 n^{2}+464 n-636\right)}{24\left(n^{2}-1\right)(n-6)(3 n-8)} I J-\frac{1}{8\left(n^{2}-1\right)} Z+\frac{1}{8(n+1)} I^{2}, \\
\bar{F}= & \frac{-\left(2 n^{3}-58 n^{2}+410 n-744\right)}{24\left(n^{2}-1\right)(n-6)(3 n-8)} I J-\frac{1}{8\left(n^{2}-1\right)} Z-\frac{1}{4\left(n^{2}-1\right)} I^{2} . \\
& \\
& \\
&
\end{aligned}
$$

We will finally require $I$ and $J$ :

$$
\begin{aligned}
& I=\pi^{2} \Gamma\left(2-\frac{1}{2} n\right) B\left(\frac{1}{2} n-1, \frac{1}{2} n-1\right)=\frac{2}{4-n}-\gamma_{\mathrm{E}}+\mathrm{O}(n-4), \\
& J=\pi^{2} \frac{\Gamma(4-n)}{\Gamma\left(3-\frac{1}{2} n\right)} B\left(n-3, \frac{1}{2} n-1\right)=\frac{1}{4-n}-\gamma_{\mathrm{E}}+\frac{3}{2}+\mathrm{O}(n-4)
\end{aligned}
$$


Note that here we have taken the surface area of the unit sphere in $n$ dimensions to be $2 \pi^{2} / \Gamma\left(\frac{1}{2} n\right)$. It is easy to see that the conventional choice of $2 \pi^{n / 2} / \Gamma\left(\frac{1}{2} n\right)$ just changes the definition of $\gamma$ [eq. (4.1)] without affecting the final results.

\section{References}

[1] S. Ferrara and B. Zumino, Nucl. Phys. B87 (1975) 207

[2] T. Curtright, Phys. Lett. 71B (1977) 185

[3] L.F. Abbott, M.T. Grisaru and H.J. Schnitzer, Phys. Rev. D16 (1977) 2995; Phys. Lett. 71B (1977) 161

[4] T. Hagiwara, S.Y. Pi and H.S. Tsao, preprint C00-22328-267 (1978) unpublished

[5] M. Grisaru, in Recent developments in gravitation (Cargese, 1980) ed. M. Levy and S. Deser (Plenum Press)

[6] H. Nicolai and P.K. Townsend, Phys. Lett. 93B (1980) 111

[7] J. Collins, A. Duncan and S.D. Joglekar, Phys. Rev. D16 (1977) 438; N.K. Nielsen, Nucl. Phys. B120 (1977) 212

[8] S.L. Adler and W.A. Bardeen, Phys. Rev. 182 (1969) 1517;

S.L. Adler, R.W. Brown, T.F. Wong and B.-L. Young. Phys. Rev. D4 (1971) 1787;

S.L. Adler, in Lectures on elementary particles and quantum field theory (Brandeis 1970) ed. S. Deser, M. Grisaru and H. Pendleton

[9] O. Piguet and K. Sibold, Nucl. Phys. B196 (1982) 428,447

[10] D.R.T. Jones and J.P. Leveille, Phys. Lett. B, to be published

[11] W.A. Bardeen, Proc. 16th Int. Conf. on High-energy physics, ed. J.D. Jackson and A. Roberts (1972);

K. Fujikawa INS-Rep.-427 (1981)

[12] M. Chanowitz, Phys. Rev. D9 (1974) 503

[13] G. 't Hooft and M. Veltman, Nucl. Phys. B44 (1972) 189

[14] J.F. Ashmore, Nuovo Cim. Lett. 4 (1972) 289

[15] C.G. Bollini and J.J. Giambiagi, Nuovo Cim. 12B (1972) 20

[16] W. Siegel, Phys. Lett. 84B (1979) 193

[17] M. Chanowitz, M. Furman and I. Hinchliffe, Nucl. Phys. B159 (1979) 225

[18] D.M. Capper, D.R.T. Jones and P. van Nieuwenhuizen, Nucl. Phys. B167 (1980) 479;

P. Majumdar, E. Poggio and H. Schnitzer, Phys. Rev. D21 (1980) 2203

[19] M.K. Fung, D.R.T. Jones and P. van Nieuwenhuizen, Phys. Rev. D22 (1980) 2995

[20] W. Siegel, Phys. Lett. 94B (1980) 37

[21] L.V. Avdeev, G.A. Chochia and A.A. Vladimirov, Phys. Lett. 105B (1981) 272

[22] D.A. Akyeampong and R. Delbourgo, Nuovo Cim. 17A (1973) 578; 18A (1973) 94; 19A (1974) 219

[23] G. Bonneau, Phys. Lett. 96B (1980) 147; Nucl. Phys. B171 (1980) 477; B177 (1981) 523

[24] P. Breitenlohner and D. Maison, Comm. Math. Phys. 52 (1977) 11

[25] D.M. Capper, Queen Mary College preprint QMC-79-17, unpublished

[26] P.K. Townsend and P. van Nieuwenhuinzen, Phys. Rev. D20 (1979) 1932

[27] D.R.T. Jones, Nucl. Phys. B87 (1975) 127

[28] D.R.T. Jones, Phys. Rev. D22 (1980) 3140

[29] L.V. Avdeev, O.V. Tarasov and A.A. Vladimirov, Phys. Lett. 96B (1980) 94

[30] D.Z. Freedman, private communication

[31] F.V. Tkachov, Phys. Lett. 100B (1981) 65 